\title{
Detection of Coupling Misalignment by Extended Orbits
}

\author{
Michael Monte $^{\mathrm{a}}$, Florian Verbelen ${ }^{\mathrm{a}}$, Bram Vervisch $^{\mathrm{a}, \mathrm{b}}$ \\ ${ }^{a}$ University of Ghent, Campus Kortrijk, Department of Industrial Systems and Product Design, Graaf \\ Karel de Goedelaan 5, B-8500, Kortrijk, Belgium \\ ${ }^{\mathrm{b}}$ University of Ghent, Department of Electrical Engineering, Systems and Automation, \\ Technologiepark 914, B-9052, Zwijnaarde, Belgium
}

\begin{abstract}
In this paper a 'SpectraQuest' demonstrator is used to introduce misalignment into a rotating machinery set-up. Depending on the coupling used in the set-up, angular and/or parallel misalignment can be brought in the rotating system. Traditionally, the data captured by accelerometers is transferred into the frequency domain in order to interpret the vibrations measured by the accelerometers. The frequency domain has proven its usefulness but even the time domain can come in handy to draw the right conclusions regarding to misalignment in a rotating set-up. Orbit plots display the integrated data captured by accelerometers, in order to display the movement of the rotating shaft. The influence of the misalignment and imbalance on these orbits will be discussed.
\end{abstract}

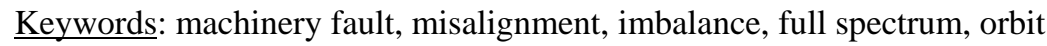

\section{Introduction}

In rotating machinery misalignment is a serious problem [1]. It can lead to stress and it can cause severe damage leading to malfunction or even breakdown. A lot of research has been done on misalignment, and is still ongoing. Several techniques have been developed to detect misalignment and other machine faults. Some techniques found in literature are vibration monitoring [2], acoustic emission [3], thermal imaging [4] and oil particle analysis [5].

The most widespread method is the use of vibration spectra. This technique is used in this paper to detect misalignment. A lot of information can be taken out of a spectrum measured by accelerometers. The time based captured data are transformed into the frequency domain and the spectra have some characteristics for detecting single faults. The problem is when several faults are in the rotating system at the same time. More advanced techniques are necessary to draw a correct conclusion based on the spectra. Full-spectra and orbits can help to detect several machine faults [6]. In this paper the orbit is extended to a third dimension. Instead of only looking at the radial direction, the axial direction also plays an important role in detecting angular and parallel misalignment.

\section{Experiments}

The set-up used for the experiments is the Machinery Fault Simulator from SpectraQuest. Three accelerometers are used to measure the vibrations, two in the radial ( $x$-horizontal and $y$-vertical) and one in the axial direction ( $z$-direction). The data captured by the three accelerometers are used to build up an extended orbit plot, a three dimensional representation of the double integrated data captured on the bearing close to the coupling where misalignment is introduced to the system. A reference measurement at a rotating speed of $25 \mathrm{~Hz}$ from an accelerometer placed horizontal ( $x$ direction) is represented in Fig. 1. These measurements were done on a well-aligned and balanced set-up. Similar measurements were done at $35 \mathrm{~Hz}$ and 50 $\mathrm{Hz}$.

Subsequently a three dimensional orbit is made from the data collected from the three accelerometers (Fig. 2). The measurements show the movement of the $1 \mathrm{x}$ vibration together with the $2 \mathrm{x}$ vibration. The third plot is the movement of the $1 \mathrm{x}$ and $2 \mathrm{x}$ vibration together. In these plots no ellipses are shown as mentioned in previous work [6], but when the regular orbits (horizontal and vertical radial direction) are plotted ellipses appear (Fig. 3). It is clear that the motion in the radial direction is very limited in this set-up (Fig. 4). 


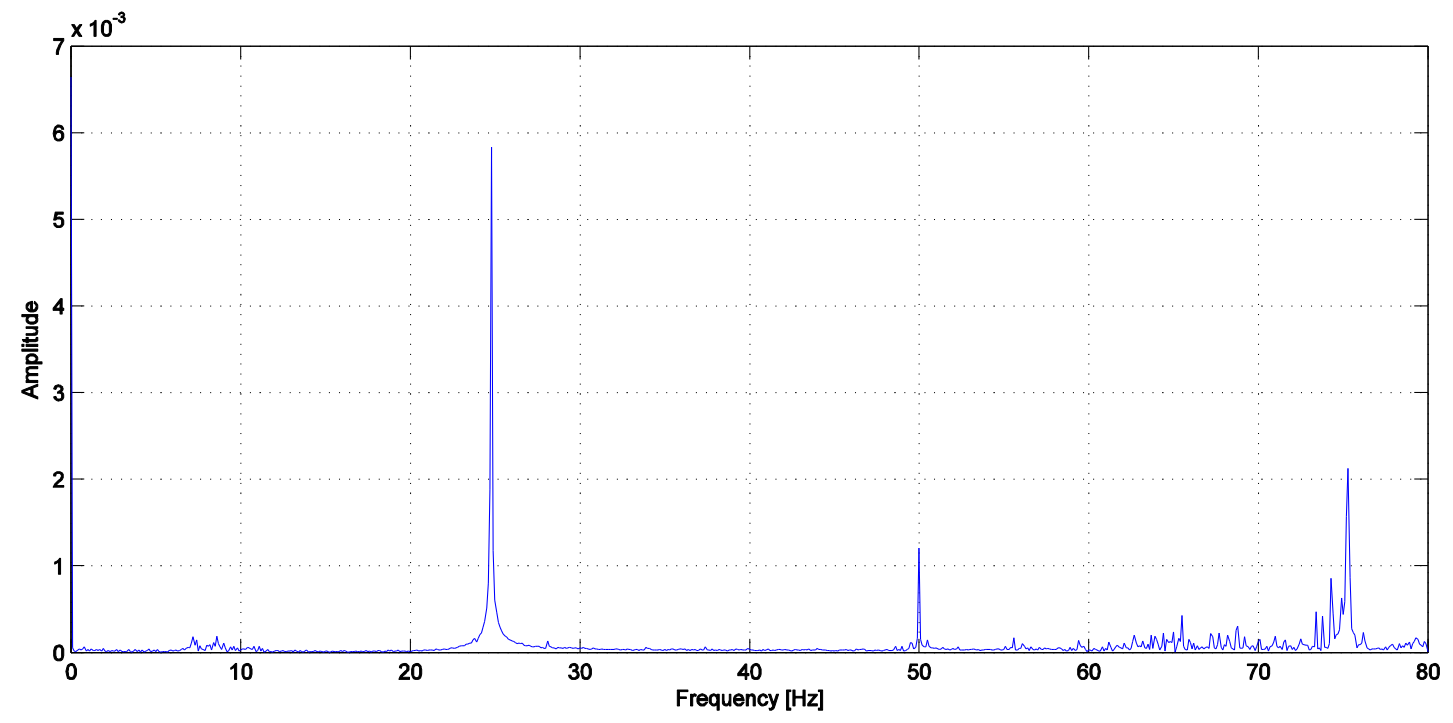

Fig. 1 Reference FFT at $25 \mathrm{~Hz}$ (horizontal)
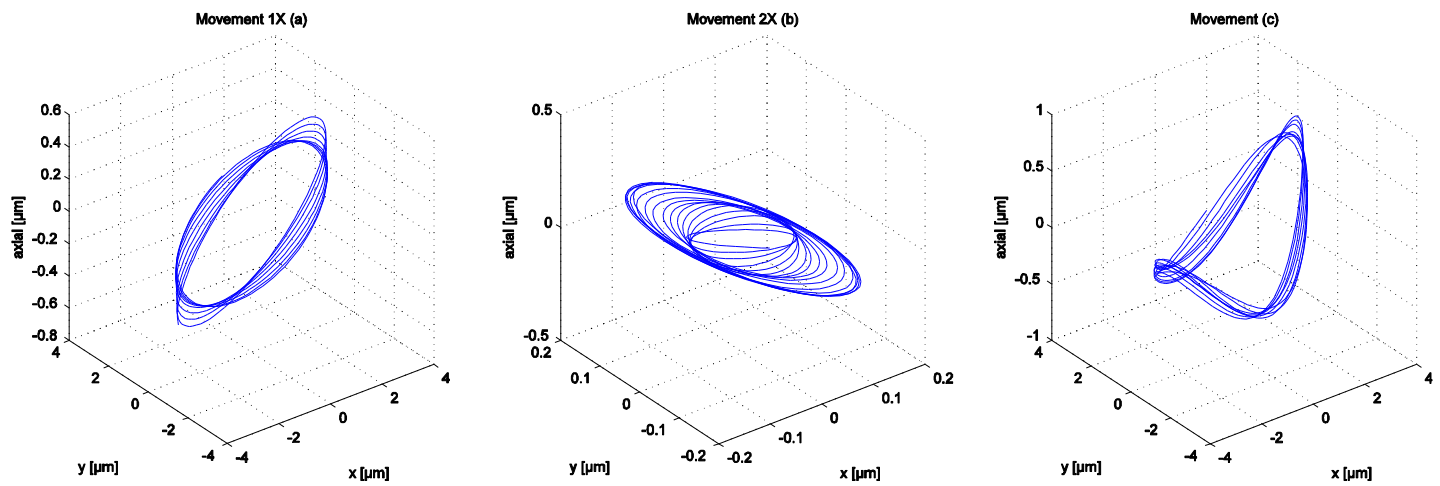

Fig. 2 Three dimensional orbit at $25 \mathrm{~Hz}$
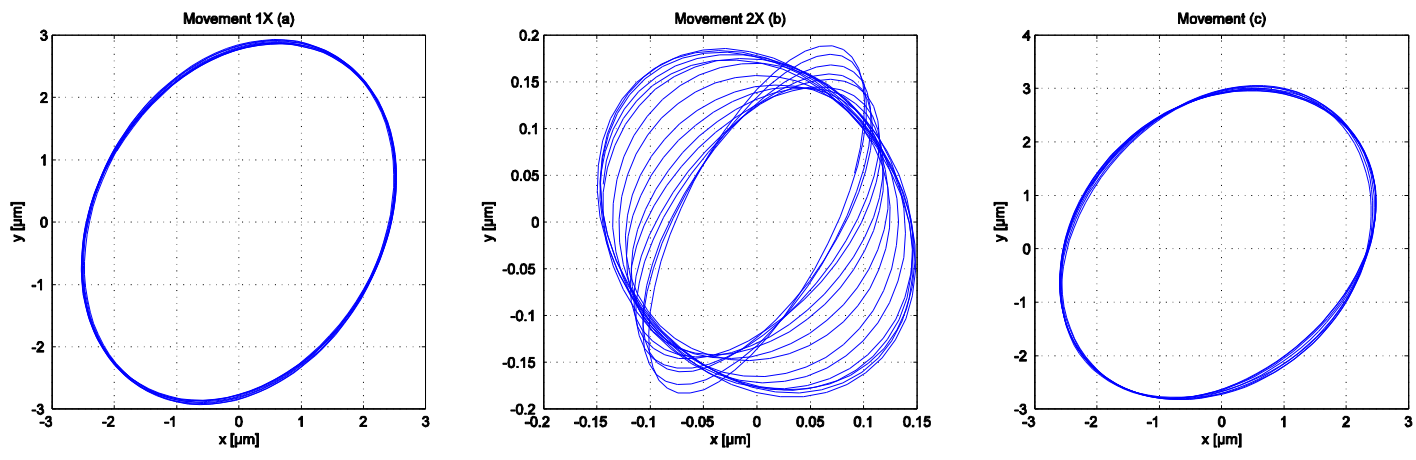

Fig. 3 Two dimensional orbit at $25 \mathrm{~Hz}$ (radial direction) 

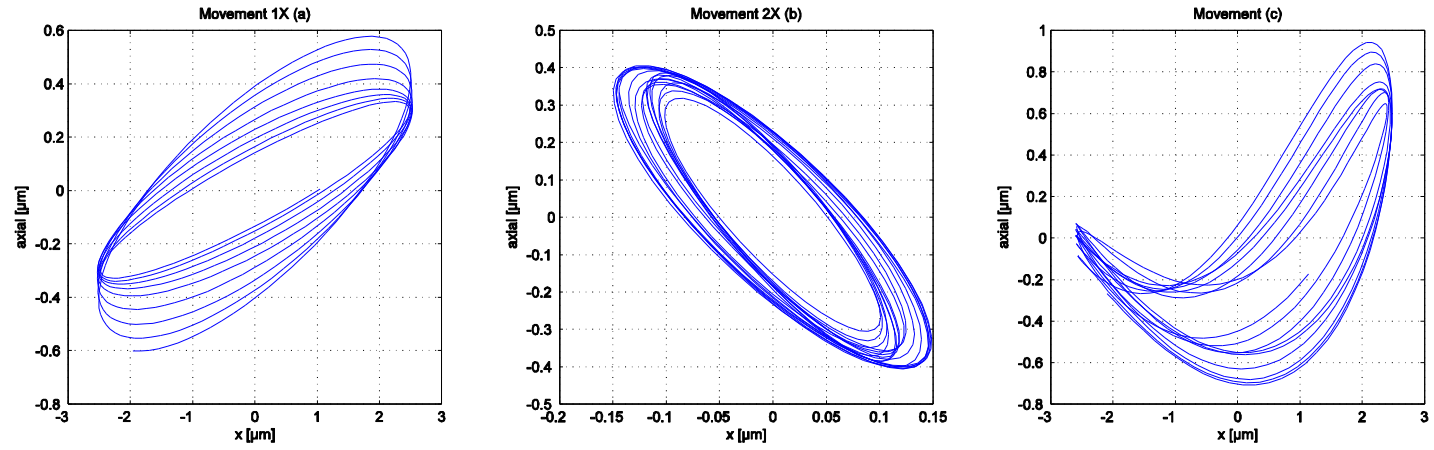

Fig. 4 Two dimensional orbit with a radial direction combined with an axial direction

\section{a. Measurements on parallel misalignment}

Next, the influence of parallel misalignment on the orbits will be discussed. On the face of it no conclusions can be made from the 3D orbit (Fig. 5). The shapes of the orbits are very similar and no more distortion is observed in the plot as the misalignment in the system is very small, but when looking at the projections in the radial and axial direction, a difference is visible so as to identify parallel misalignment (Fig. 6). The amount of motion in the vertical direction ( $y$ direction) is higher when parallel misalignment is introduced. The orbit in the axial direction is very similar compared with the orbit in a well-aligned set-up (Fig. 7). The increase at $2 \mathrm{x}$ rotational speed is according to what is mentioned in literature [7].

Movement $1 \mathrm{X}$ (a)

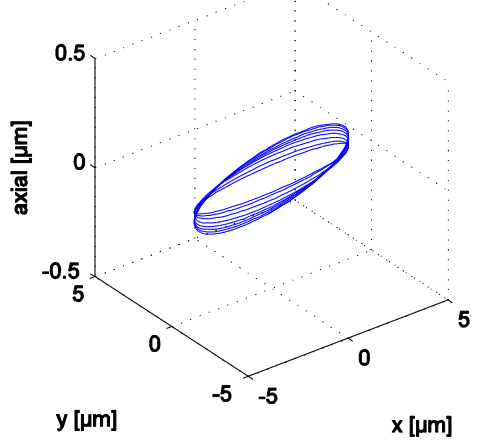

Movement 2X (b)

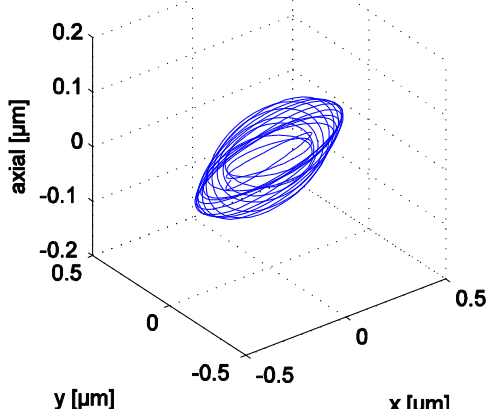

Movement (c)

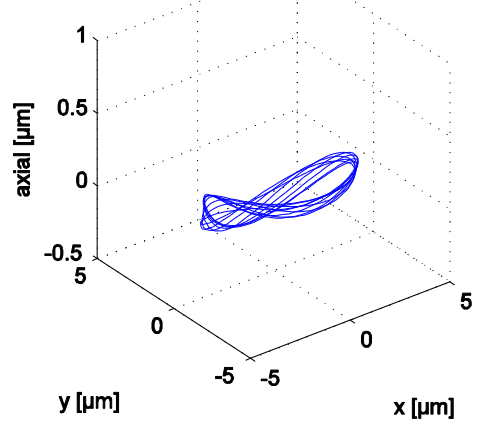

Fig. 5 Three dimensional Orbit at $25 \mathrm{~Hz}$ with parallel misalignment
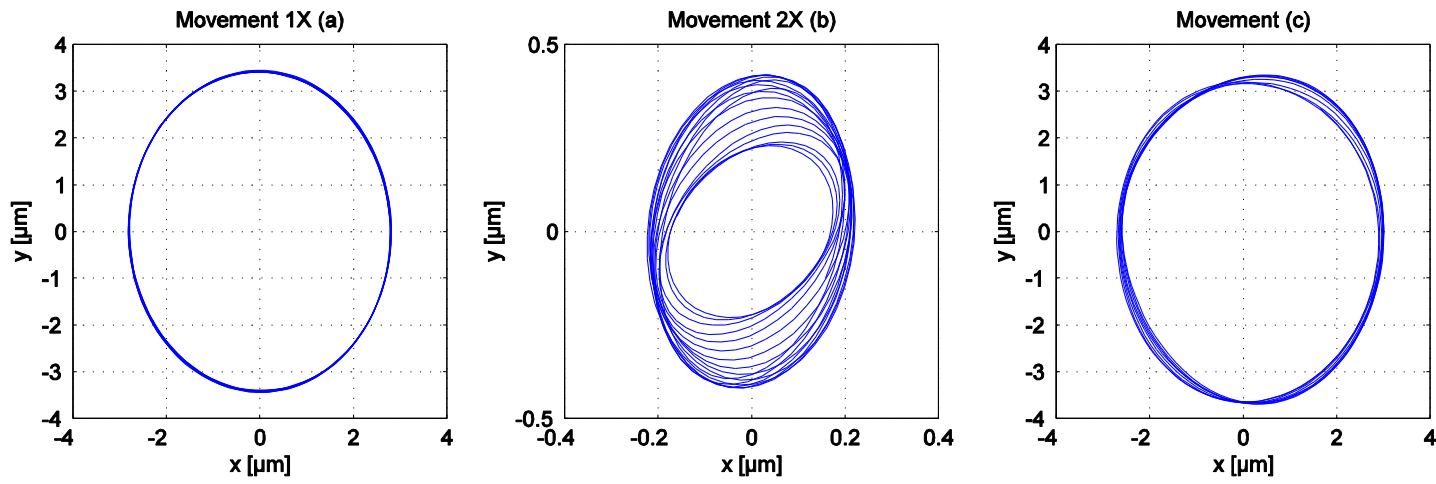

Fig. 6 Two dimensional orbit in radial direction at $25 \mathrm{~Hz}$ with parallel misalignment 

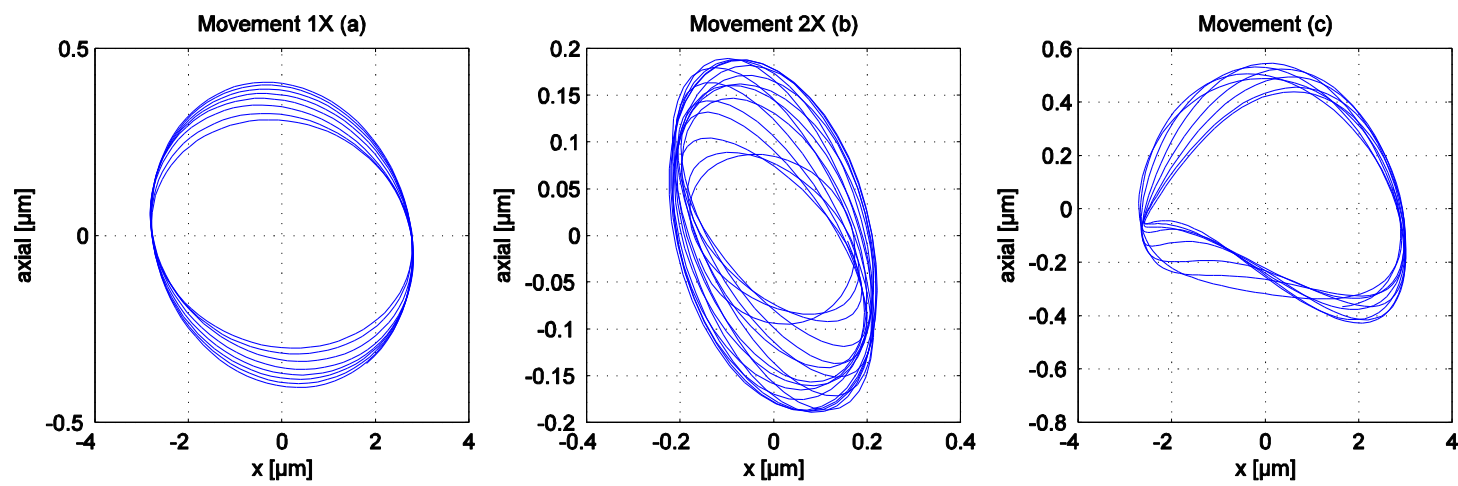

Fig. 7 Two dimensional orbit in axial direction at $25 \mathrm{~Hz}$ with parallel misalignment

\section{b. Measurements on angular misalignment}

For angular misalignment an increase in the 1x peak should be noticed. When looking at the three dimensional orbit this increase is also visible (Fig. 8). The projection in the radial direction does not reveal any increase (Fig. 9) but both projections in the axial direction exhibit an increase at 1x rotational speed (Fig. 10,11).
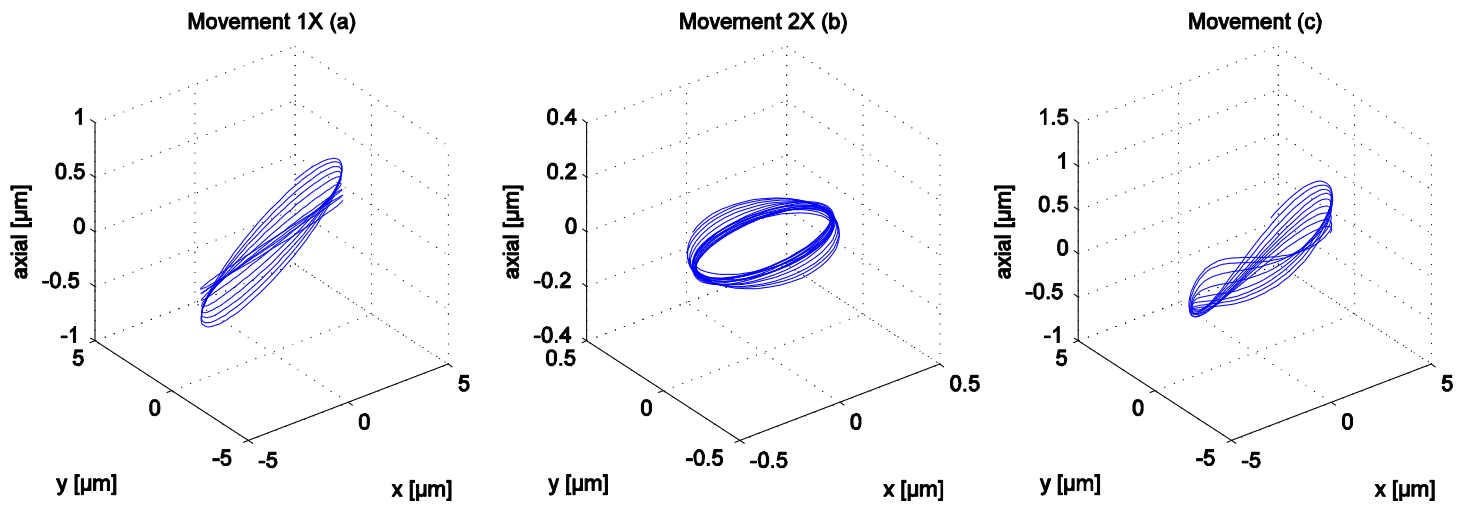

Fig. 8 Three dimensional orbit at $25 \mathrm{~Hz}$ with angular misalignment
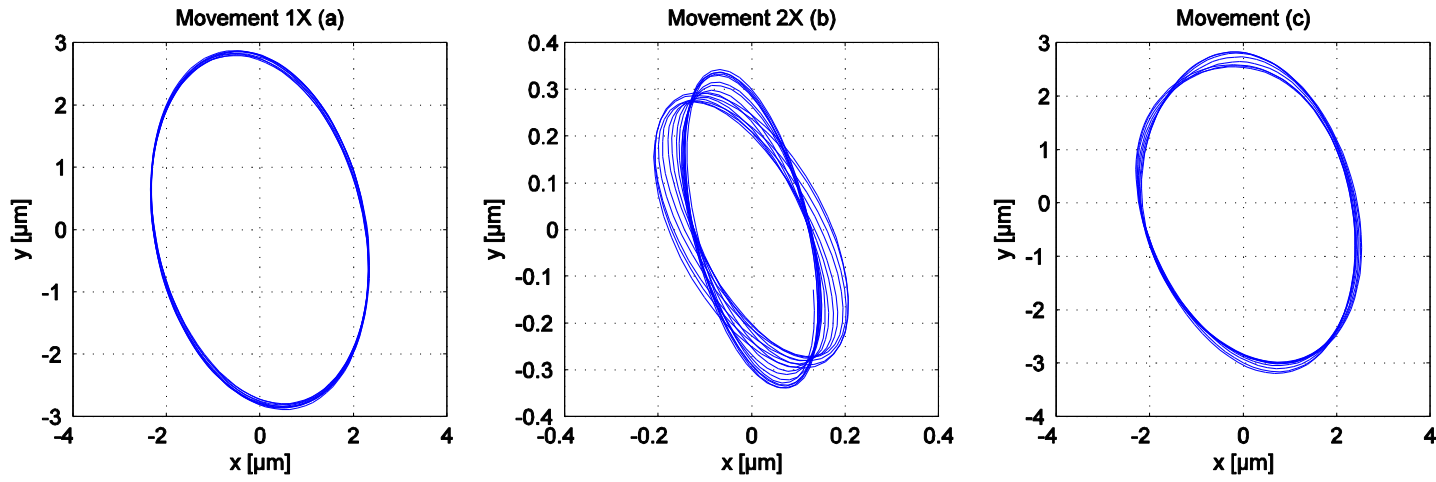

Fig. 9 Two dimensional orbit in radial direction at $25 \mathrm{~Hz}$ with angular misalignment 

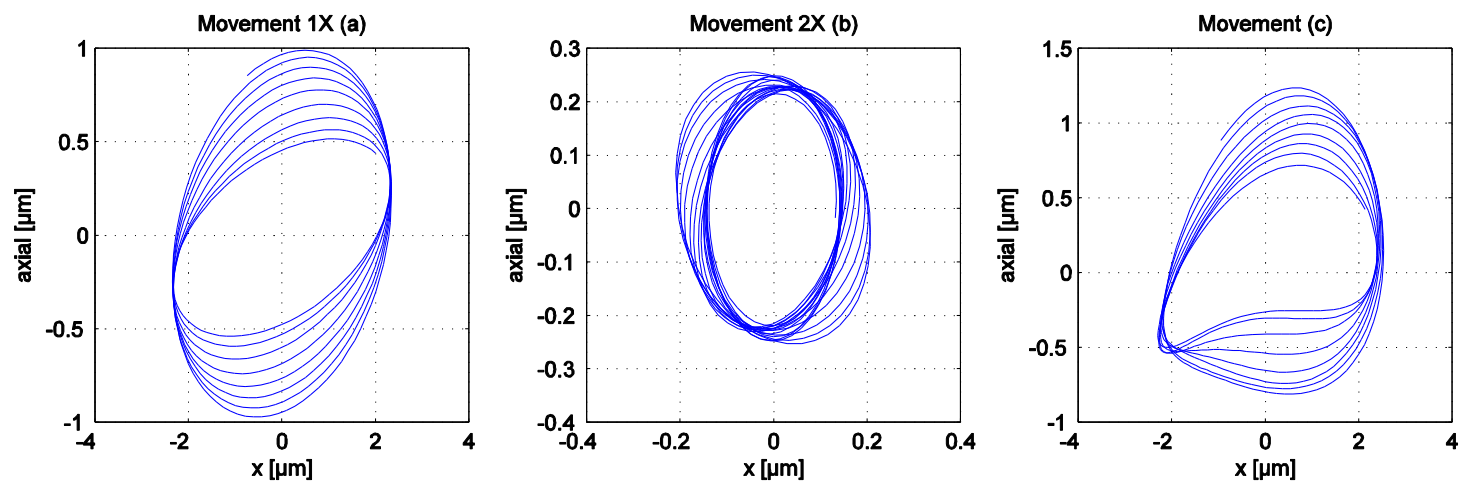

Fig. 10 Two dimensional orbit in axial direction at $25 \mathrm{~Hz}$ with angular misalignment
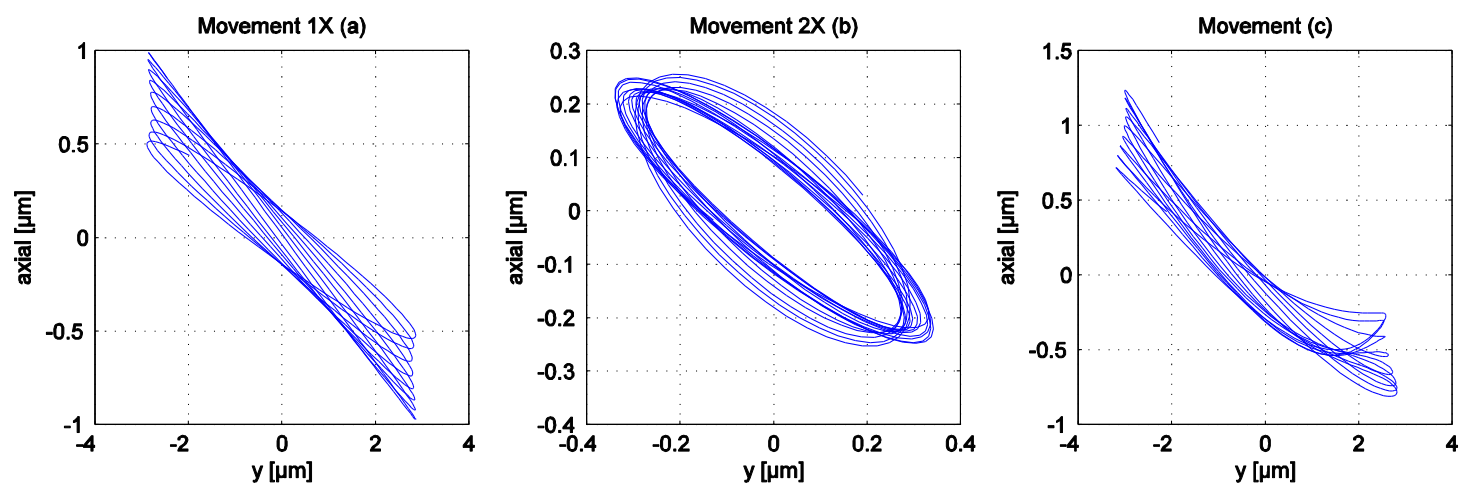

Fig. 11 Two dimensional orbit in axial direction at $25 \mathrm{~Hz}$ with angular misalignment

\section{c. Measurements with imbalance and combined faults}

The introduction of imbalance to an aligned system results in an increased amplitude of the movement at 1x rotational speed, both in axial and radial direction (Fig. 12,13,14). When parallel or angular misalignment are combined with imbalance full spectra are necessary to make the proper analysis, but with 3D Orbits it is also possible to detect the combined fault. In Fig. 15 and Fig. 16 an increase of the movement can be noticed at 2x rotational speed in radial direction. In the full spectrum of these measurements the parallel misalignment is not detected (Fig. 17). The higher peak marks the imbalance at $1 \mathrm{x}$ the rotational speed compared with $-1 \mathrm{x}$, but the peak at $2 \mathrm{x}$ rotational speed is very limited.
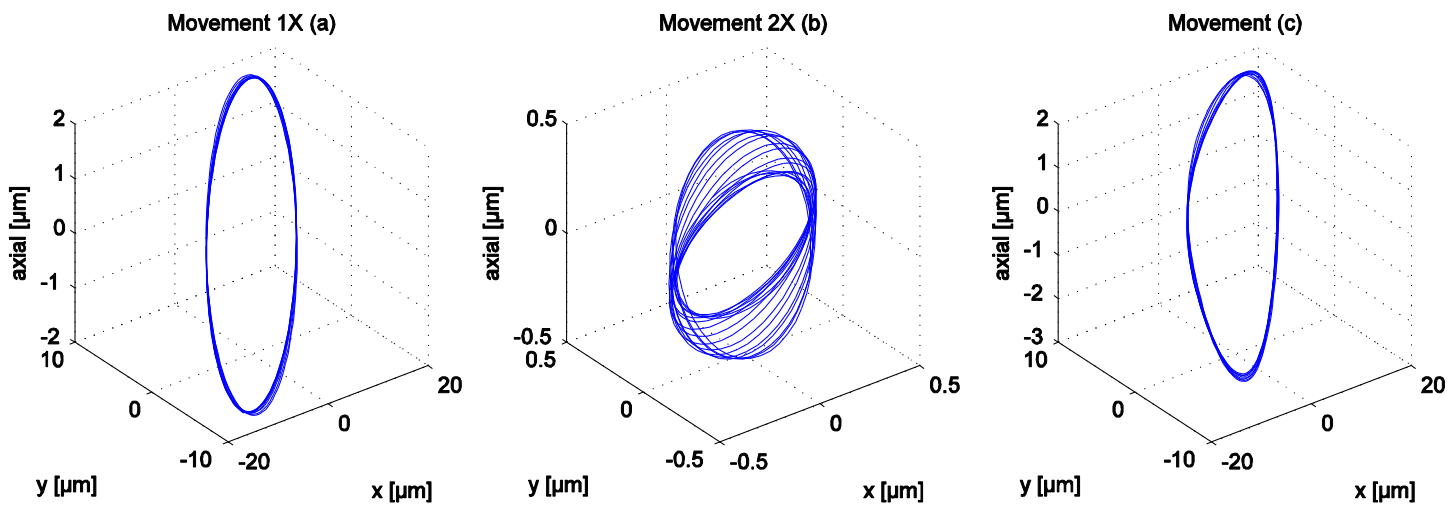

Fig. 12 Three dimensional orbit at $25 \mathrm{~Hz}$ with imbalance 

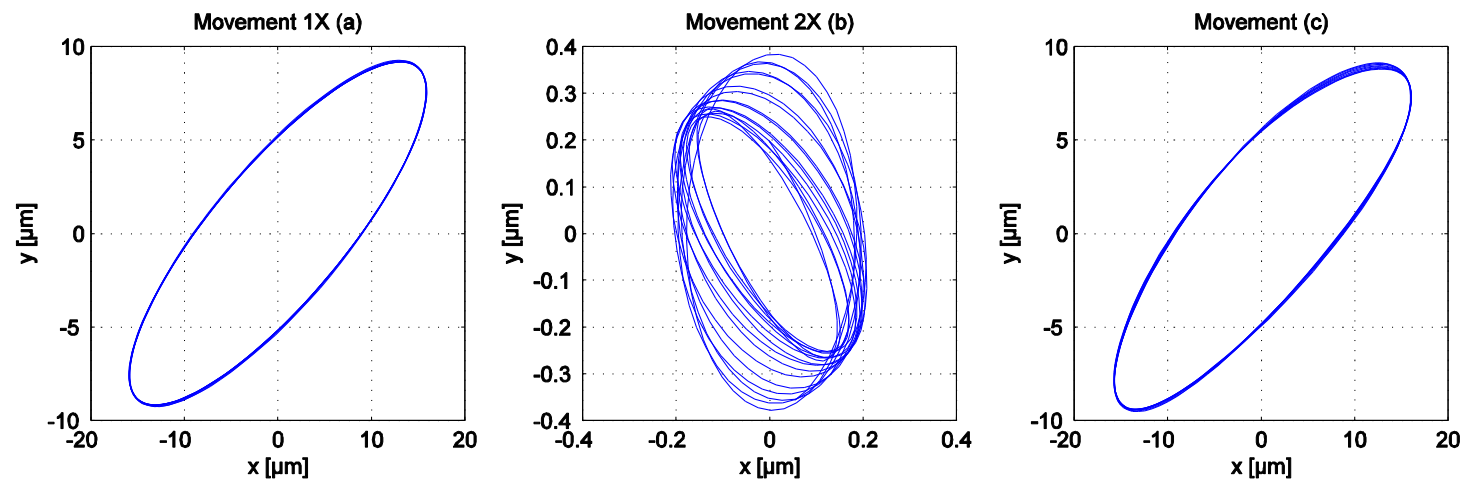

Fig. 13 Two dimensional orbit at $25 \mathrm{~Hz}$ with imbalance in radial direction
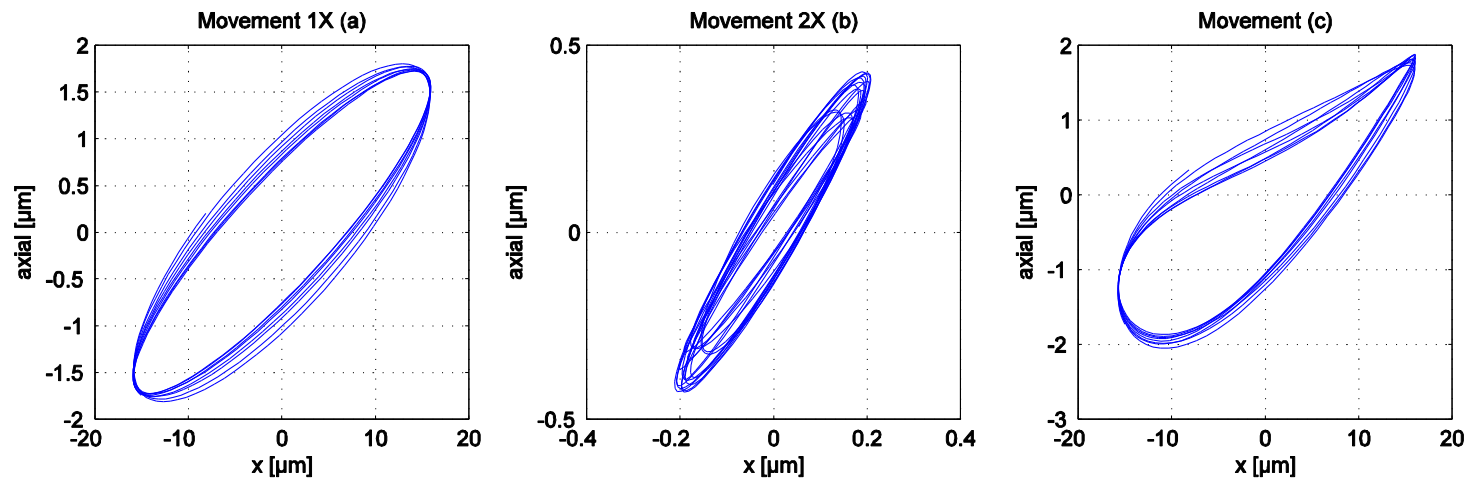

Fig. 14 Two dimensional orbit at $25 \mathrm{~Hz}$ with imbalance in radial direction
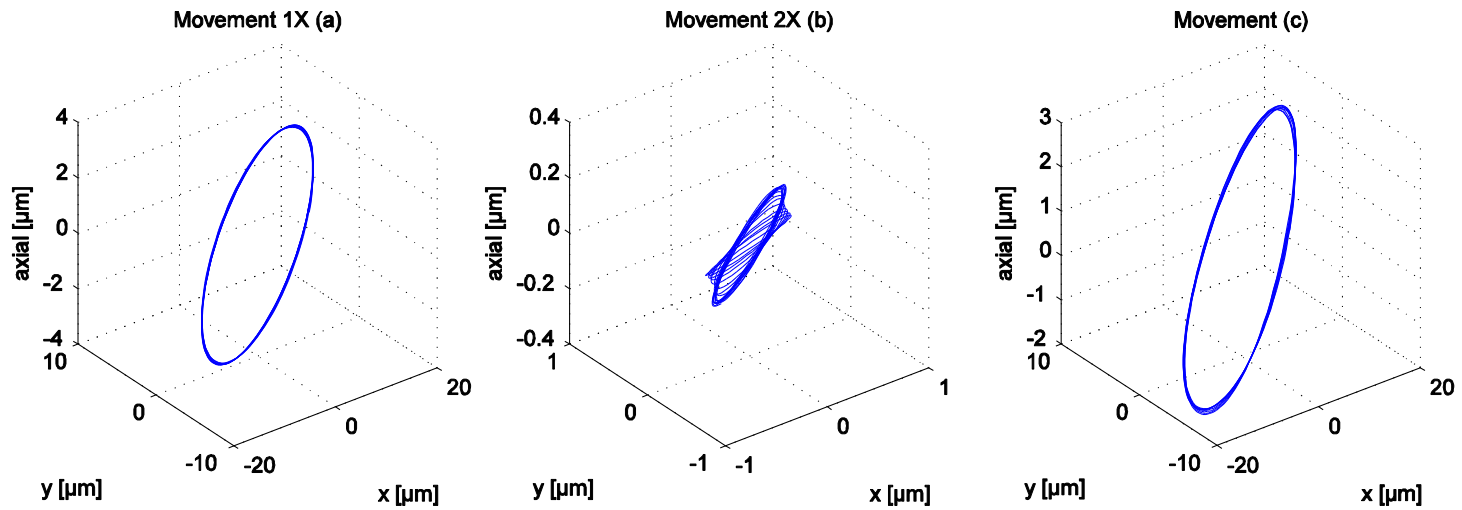

Fig. 15 Three dimensional orbit at $25 \mathrm{~Hz}$ with imbalance and parallel misalignment 

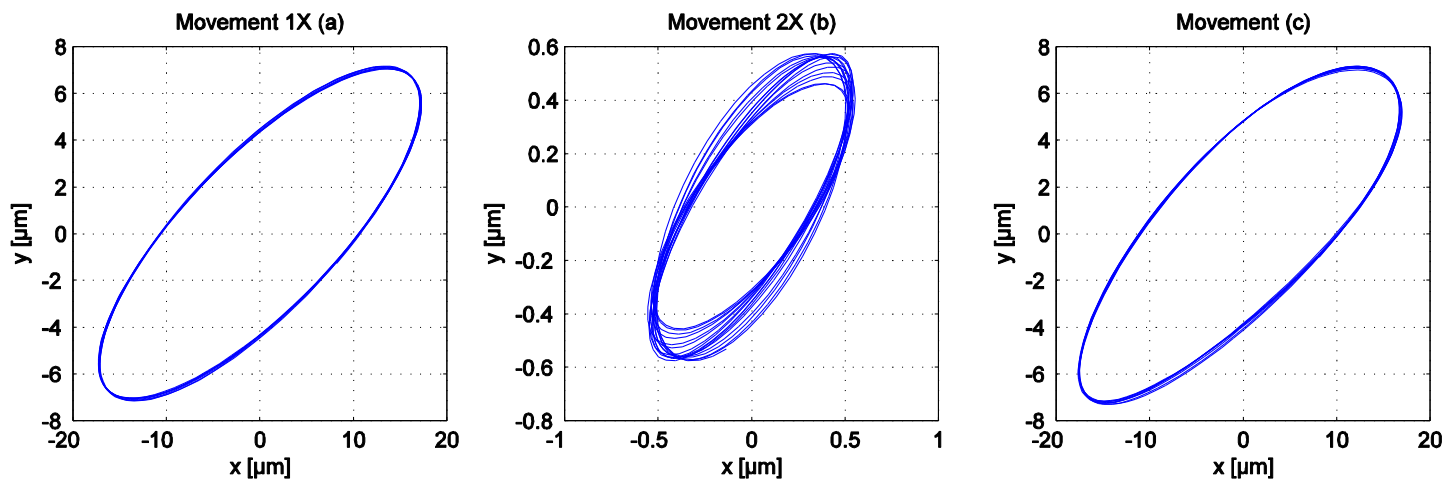

Fig. 16 Two dimensional orbit at $25 \mathrm{~Hz}$ in radial direction with imbalance and parallel misalignment

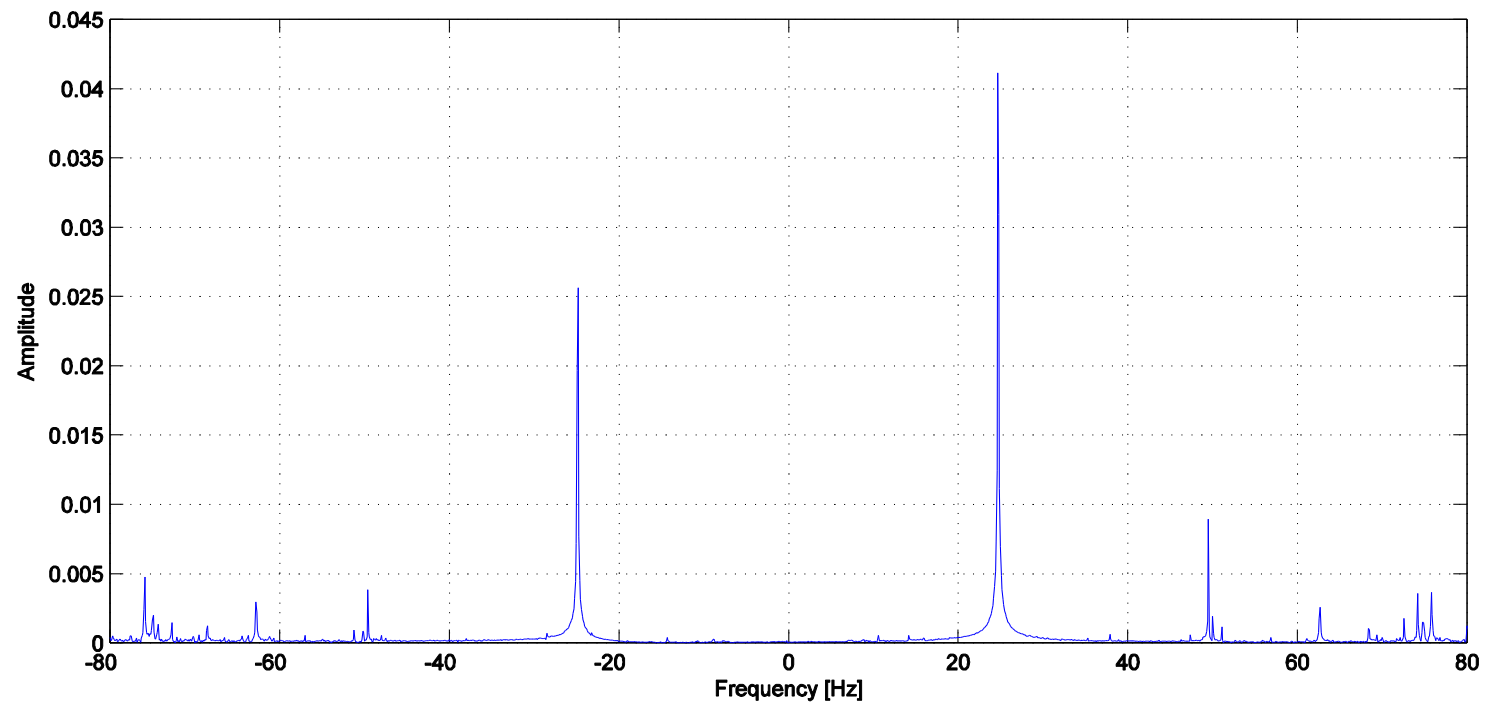

Fig. 17 Full spectrum at $25 \mathrm{~Hz}$ in radial direction with imbalance and parallel misalignment

\section{Conclusions}

Orbitals and full spectra had already proven their usefulness in detecting machinery faults from vibration measurements. But by extending the orbit plot to the axial direction more information can be taken out of the time-domain data captured by accelerometers. The difference between angular and parallel misalignment is very clear when studying the three dimensional orbits. Angular misalignment can be detected by looking at the axial direction on the 1x peak, while parallel misalignment is detected in the radial direction at the $2 x$ peak. The presence of imbalance leads to a higher excitation of the orbits. An additional advantage of three-dimensional orbits is that even a small misalignment can be detected while this is difficult to spot in a traditional spectrum.

Additional research is absolutely needed in order to confirm these findings and to compare the measurements with other couplings used in the rotating set-up. It is expected that the coupling will have a big influence on future measurements. The three-dimensional orbitals and full spectrum can be used as an additional tool when vibrational data are studied. 


\section{References}

[1] J. Piotrowski, Shaft alignment handbook. 2010.

[2] S. S. Kumar and M. S. Kumar, "Condition Monitoring of rotating machinery through Vibration Analysis," vol. 73, no. April, pp. 258-261, 2014.

[3] J. L. Ferrando Chacon, E. Artigao Andicoberry, V. Kappatos, G. Asfis, T.-H. Gan, and W. Balachandran, "Shaft angular misalignment detection using acoustic emission,” Appl. Acoust., vol. 85, pp. 12-22, Nov. 2014.

[4] C. C. Ciang, J.-R. Lee, and H.-J. Bang, "Structural health monitoring for a wind turbine system: a review of damage detection methods," Meas. Sci. Technol., vol. 19, no. 12, p. 122001, Dec. 2008.

[5] G. E. Newell, "Oil analysis cost-effective machine condition monitoring technique," Ind. Lubr. Tribol., vol. 51, no. 3, p. 119, 1999.

[6] M. Monte, F. Verbelen, and B. Vervisch, "The Use of Orbitals and Full Spectra to Identify Misalignment," in Structural Health Monitoring, Volume 5, 2014.

[7] a. S. Sekhar and B. S. Prabhu, "Effects of coupling misalignment on vibrations of rotating machinery," J. Sound Vib., vol. 185, no. 4, pp. 655-671, Aug. 1995. 